\title{
Assessing Risks to Inform Resilience: a Criticality Assessment of the British Railway Network
}

\author{
Dr Raghav Pant ${ }^{1}$, Dr Simon Blainey ${ }^{2}$, Prof Jim Hall ${ }^{3}$ and Prof John Preston ${ }^{4}$ \\ ${ }^{1}$ Environmental Change Institute, University of Oxford, UK, \\ ${ }^{2}$ Faculty of Engineering and the Environment, University of Southampton, UK, \\ ${ }^{3}$ Environmental Change Institute, University of Oxford, UK, \\ ${ }^{4}$ Faculty of Engineering and the Environment, University of Southampton, UK
}

\begin{abstract}
National infrastructures are constantly at risk from extreme weather events and random shocks that induce widespread failures across such systems. For example, the vulnerability of Britain's national-scale rail infrastructure has been highlighted during extreme floods, and it seems likely that the levels of risk faced by transport infrastructures more generally will increase in future years. Given the importance of rail infrastructure, there is a clear need for an improved understanding of the risks which it faces. Such a risk assessment would aim to identify the relative criticality of the network elements which different risk events are likely to affect, leading towards better risk management and resilience investment decisions. This paper provides a methodology to meet this need, by analysing the systemic risk to Britain's rail infrastructure from a range of disruptive events. It first considers the range of events and processes which have the potential to disrupt operation of the rail network. Alongside this, a procedure is developed for assessing the relative criticality of different nodes and edges on the network based on the passenger traffic they carry. Two case study risk types (floods and traction system failures) are used to demonstrate how criticality assessment can identify those parts of the rail network which are most at risk of causing substantial disruption to rail traffic, and therefore are most critical to maintaining national mobility. The paper concludes by considering the implications of this analysis for investment decisions and the potential for transferring this methodology to other spatial or economic contexts.
\end{abstract}

Keywords: Resilience, Risk Assessment, Railway

\section{BACKGROUND AND OVERVIEW:}

Coastal flooding in early 2014 brought the issue of infrastructure risk into the media spotlight, when the only rail link to a large area of South-West England was severed for several months after tracks and their protecting sea wall were washed away by a winter storm. Attention was focused on the question of whether or not it was desirable for transport networks to be reliant on single 'critical' network edges (links), particularly when such edges are exposed to a high level of risk. The floods also led to a more general debate on the level of risk from and resilience to coastal flooding across the UK rail network, set against the background of continuing climate change which suggests that the frequency of coastal flooding events will increase over the $21^{\text {st }}$ century. While coastal flooding has the potential to cause serious problems, it is not certain that it is necessarily the most serious risk factor facing Britain's railway infrastructure or that the edges affected (and therefore potentially targeted for investment to increase resilience) are in fact the most critical edges on the network.

The relative criticality of different network edges depends both on current patterns of rail services and rail travel and on future demographic changes to these patterns. There are also clear interdependencies with energy infrastructure, 
without which mobility at current levels would be impossible. Research has shown that the most likely demographic future for the UK will involve sustained population growth, potentially to over double the current level ${ }^{1}$. While evidence suggests that growth in mobility per capita may be levelling off amongst some socio-economic groups ${ }^{2}$, it still seems likely that the growing population of Britain will expect to enjoy at least equivalent levels of mobility to those available to British residents today. This means transport infrastructure systems will need to accommodate much greater aggregate travel volumes than is currently the case.

There are though significant resource implications of this growth in travel, as it has the potential to conflict with the urgent requirement to reduce transport-related carbon emissions, which made up 27\% of the UK total in 2011

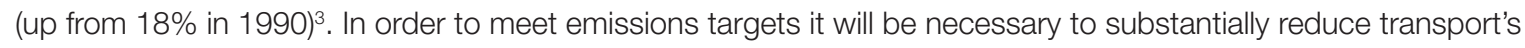
carbon emissions by shifting travellers to the most environmentally-friendly modes possible for particular journeys. Despite predicted fuel efficiency gains for road ${ }^{4}$ and air ${ }^{5}$ transport, rail is still expected to maintain an environmental advantage, meaning that mode shift to rail should be encouraged. However, while such mode shift to rail could help to meet the needs of a larger population using fewer resources, this will mean placing more stress and more economic 'responsibility' on the existing railway infrastructure. In a situation where the rail network is responsible for a greater proportion of national mobility, major asset failures such as those seen in Devon in early 2014 will have a correspondingly greater economic and social impact. Breakdowns of critical infrastructures disrupt essential services, which have serious consequences including severe economic damage, grave social disruptions or even large-scale loss of life $e^{6,7,8}$. There is thus a pressing need for an improved understanding of the risks which face the rail network and of the relative criticality and substitutability of the edges and nodes which these risk events would impact on. This paper provides a methodology to meet this need, by analysing the systemic risk to Britain's rail infrastructure from a range of disruptive events.

\section{RISKS FACING THE RAIL NETWORK}

Risks to the rail system are defined as events or processes which have the potential to render certain edges or nodes on the rail network partially or fully inoperable. They may take place at a range of spatial and temporal scales, and both the magnitude and the duration of their impacts can vary widely. While hydrological risks such as coastal, river and groundwater flooding have enjoyed a high priority in recent months, they are just one part of a wide-ranging set of environmental risks which can also include heatwaves (causing track buckling), problems caused by ice or snowfall, and landslips and slope failures caused by either saturation or drought. Structural failure of elements of the rail infrastructure can result from the poor quality of construction or maintenance of particular assets, and this can relate both to large scale structures such as tunnels and viaducts and to smaller components such as rails (with the derailment at Hatfield in 2000 caused by a broken rail being a notorious example of the latter). Operating systems such as signals or points are also prone to occasional failures, and while the 'fail-safe' nature of such systems means that these failures are unlikely to cause accidents, they can still cause significant short-term disruption to operations, as can train failures. Human actions (both intentional and accidental) can also lead to disruption, for example as a result of bridge strikes and level crossing collisions (both examples of road user failures impacting on the rail system), suicides and terrorist attacks. While extensive research has been carried out to quantify the level of many of these risks, the wide range of risk events combined with the variation in risk likelihood over space means that assessment of the aggregated risk of disruption for a particular node or edge can be very complex.

1 Tran, M. et al. National Infrastructure Assessment: Analysis of Options for Infrastructure Provision in Great Britain. Environmental Change Institute, University of Oxford (2014).

2 Le Vine, S., Jones, P. On the move: making sense of car and train travel trends in Britain, RAC Foundation (2012).

3 Department for Transport. Transport Statistics Great Britain 2013, DfT, London (2013).

4 Brand, C. UK Transport Carbon Model: Reference Guide. Working Paper. Environmental Change Institute, University of Oxford (2010).

5 Schafer, A. et al. TOSCA Project Final Report: Description of the Main S\&T Results/Foregrounds, EC FP1 Project (2011).

6 Cabinet Office. Strategic framework and policy statement on improving the resilience of critical infrastructure to disruption from natural hazards. Whitehall, London, UK (2010).

7 Homeland Security. NIPP 2013: Partnering for Critical Infrastructure Security and Resilience (2013).

8 ICE. The State of the Nation: Defending Critical Infrastructure. Institution of Civil Engineers, London, UK (2009). 


\section{ASSESSING RELATIVE IMPORTANCE OF EDGES AND NODES}

Just as the likelihood of risk occurrence varies hugely over space, the impact of risk occurrence also varies substantially from place to place, particularly with regard to the total disruption caused to the operation of the rail network and therefore to national mobility. For example, with the British rail infrastructure a risk event which halts all rail operations on a branch line in the Scottish Highlands will have a much smaller disruptive impact than an event of similar magnitude which affects a major commuter route into London. It should be noted that the volume of traffic on a particular route is not the only determinant of aggregate disruption, as in a dense network where diversionary routes are available the loss of a particular link will have a much smaller impact than it would if that edge formed the only route between two large traffic generators/attractors.

In order to assess the total impact of risk events in particular locations, a methodology has been developed to estimate railway network flows to a high level of spatial disaggregation. Flows are estimated at nodes (stations or junctions) and edges (tracks) through which we can infer the relative importance of certain travel patterns over others, leading towards a network criticality assessment. In this study the relative importance of the railway network assets (nodes and edges) is evaluated in terms of the daily usage of the network by passengers travelling along different routes. We have built an origin-destination trip assignment model for Britain's railway network in order to develop estimates for network vulnerability due to any external shock impacts. Later in the vulnerability assessment being done here we are interested in quantifying the disruptive impacts on passenger travel due to removal of affected stations, junctions or track sections.

This railway trip assignment model uses two datasets, namely: (i) The Office of Rail Regulation's (ORR) station usage dataset, which provides details of the annual number of passengers entering, exiting and interchanging at all railway stations in Britain ${ }^{9}$, and (ii) timetable data from the Association of Train Operating Companies (ATOC), which gives full details of routing and calling points for all passenger trains operating on the British rail network ${ }^{10}$. From these datasets the trip assignment model calculates the daily number of passenger trips between stations (or junctions) along specific routes (tracks) using the following steps:

1. From the train timetable data we select a representative weekly schedule and calculate the daily number of trains at each station divided into number of trains originating, intermediate or terminating at the station.

2. Using the station usage data and the step 1 estimates, we calculate the daily number of passengers using stations by converting the annual estimates into weekly estimates and then assigning these estimates to particular days in proportion to daily train frequencies. We perform separate calculations for passenger entries plus interchanges, and passenger exits plus interchanges.

3. Each train schedule in the timetable data indicates the trip information for a particular train. For each trip in the timetable we estimate all the station pairs where passengers board and alight (origin-destination (O-D) pairs).

4. The daily station usage estimates (step 2) have to be disaggregated into flows along specific paths. We define a trip attractiveness factor for each entry station, which is the product of the number of trains along the path and the volume of passengers at the exit (plus interchange) stations along the path. A path with a higher trip attractiveness factor will attract more passengers from the entry stations. At each entry station we convert the station usage estimates into trip entry estimates by dividing in proportion to the trip attractiveness factor.

5. Once we have trip entry estimates we calculate the trip exit estimates, which give the number of exits at each O-D pair along a path. We assume that along a path the numbers of passengers getting off at stations are in direct proportion to the station's usage.

6. The methodology used in steps 4 and 5 guarantees that for a chosen path the sum of trip entry estimates at all stations is equal to the sum of trip exit estimates at all stations. Hence the flow is conserved for each trip assignment.

7. The final outcome of the calculations results in estimates of the number of the passenger-trips between $O-D$ station pairs along specific routes on the rail network.

Figure 1(a) illustrates the results of the trip assignment analysis, showing the size of the daily passenger flows (for an average working weekday) across the British rail network. The railway network is comprised of 2539 stations, 1420

9 ORR. Office of Rail Regulation: Estimates of Station Usage. UK. Available at: http://www.rail-reg.gov.uk/server/show/nav.1529 (2013).

10 ATOC. Association of Train Operating Companies: Timetable data. UK. Available at: http://data.atoc.org/(2013). 
junctions and 4457 tracks (edges). These results indicate the aggregated daily travel patterns for the railway network and can be used to assess the relative importance or criticality of individual stations, junction or track sections in the railway network. The relative criticality of different edges is shown in Figure 1(b), where the different edges are ranked based on the fraction of total passenger trips assigned to them. Figure 1(a) provides a spatial description of the network criticality and Figure 1(b) result shows that a relatively small number of edges (mainly located in and around London) have very large flows compared to the rest of the network.
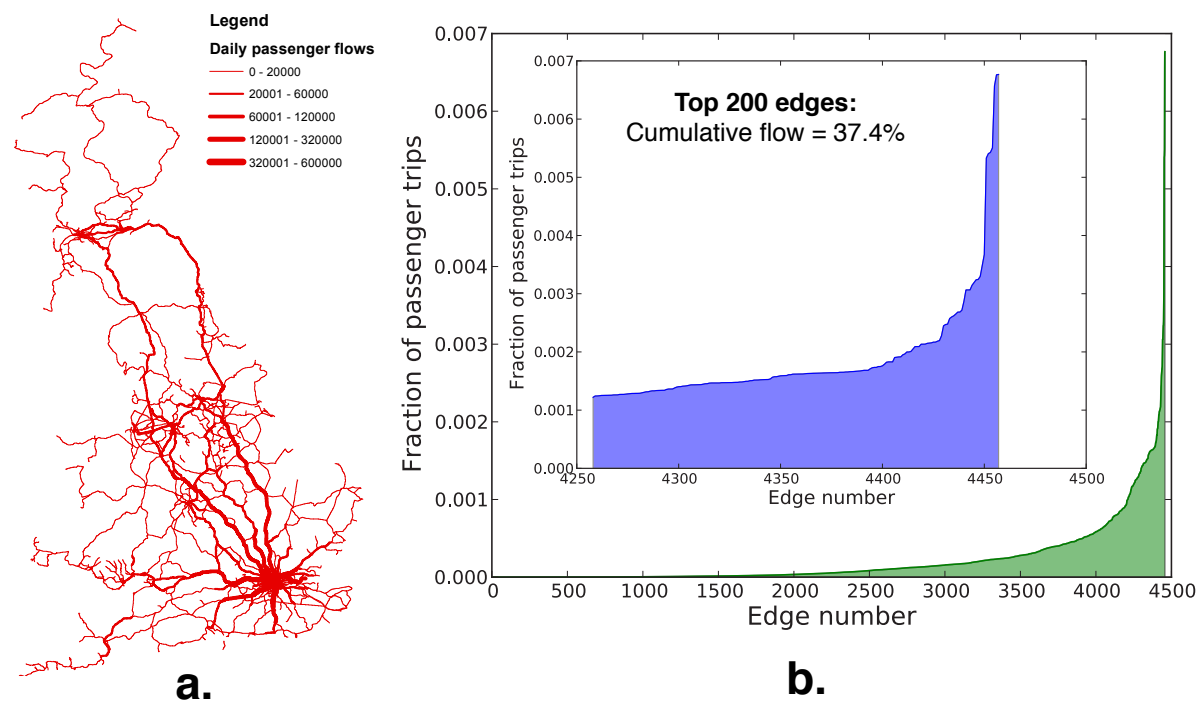

b.

Figure 1: (a) Results of trip assignment analysis showing estimates of daily number of passenger trips across individual railway network edges on the GB rail network. (b) Distribution of the passenger flows (expressed as fractions of total flows) showing the relative importance of a small number of edges.

\section{ASSESSING RISK TO LINKS AND NODES}

Using the Section 3 criticality assessment results for different nodes and edges in the railway network we can understand the possible failures that induce the greatest risks to the railway network. As discussed in Section 2 there are multiple failure scenarios that induce risks on the railway network. In the current analysis we explore two possible risk scenarios for the railway network: (i) flooding of stations and junctions, and (ii) failures of the energy supply system that provides traction electricity for specific routes.

For flood vulnerability assessment we have used the National Flood Risk Assessment (NaFRA) flood likelihood map for England and Wales ${ }^{11}$ (Environment Agency, 2009), which provides information on the estimated likelihood of flooding to areas of land within the flood plain of an extreme flood $(0.1$ per cent or 1 in 1000 chance of fluvial and/or tidal flooding in any year). The analysis shows that 543 out of 3959 nodes in the rail network lie in areas where there is some likelihood of flooding during a 1 in 1000 year flood event. Figure 2(a) shows the distribution of the passenger trips (expressed as fractions of the total network trips) through the nodes and their associated edges. Compared with Figure 1(b), the result indicates that some of the very high flow network routes are at risk of flooding and hence most vulnerable. For risk planning and improved network resilience appropriate flood defences or other resources can be allocated towards these high flow routes.

Figure 2(b) shows the energy supply system that provides traction electricity for specific routes. It consists of 125 substations (shown as nodes in the inset Figure 2(b)). The result in Figure 2(b) shows multiple passenger trip disruption outcomes when certain fractions of the traction substations are considered failed (or removed), thereby cutting off the electricity supply to the rail network. Most of the traction systems are located along routes where the majority of the passenger flows occur, which results in almost $90 \%$ of trips lost if the entire electric traction system shuts down.

11 Environment Agency. Flooding in England: A National Assessment of Flood Risk. Bristol, UK (2009). 


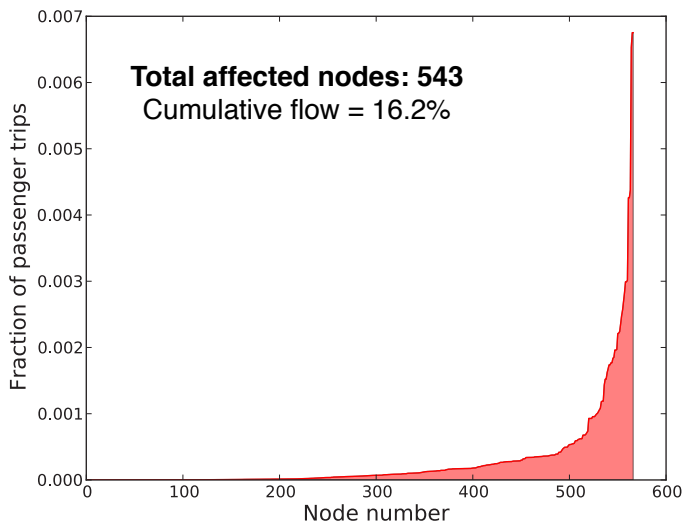

a.

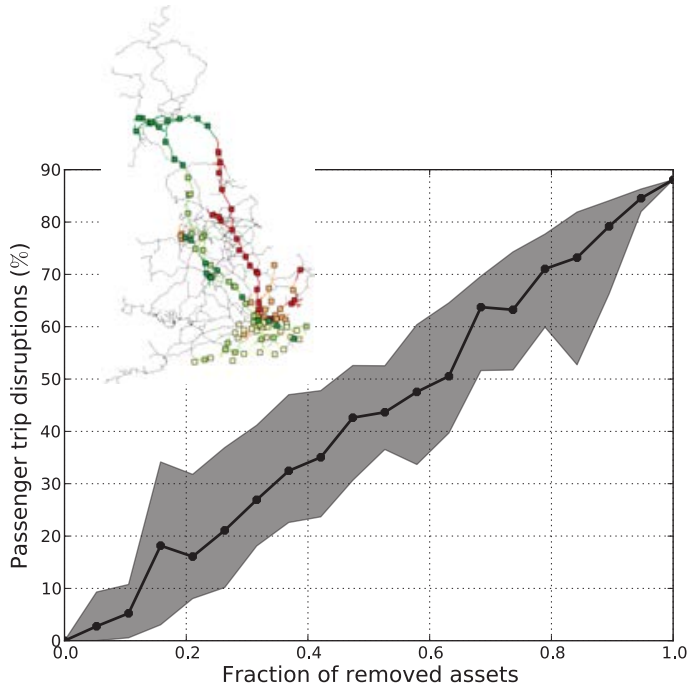

b.

Figure 2: (a) The distribution of the passenger trips at risk due to nodes (and corresponding edges) that are vulnerable to 1 in 1000 year flood event, and (b) Vulnerability of the overall rail network due to traction system (shown in the inset figure) failures.

\section{IMPLICATIONS}

This paper has demonstrated how the measure of infrastructure criticality developed here allows identification of those links and nodes which are most vulnerable to a range of risks, and which therefore have the greatest potential to adversely affect the railway network's ability to enable the effective functioning of the systems with which it shares interdependencies. When extended to cover all risk categories it could therefore be used as a tool to assess where investment in increased resilience should be focused, whether through measures to reduce the likelihood of risk events occurring, to mitigate the effects of such risks when they do occur, or to provide diversionary routes to increase the resilience of the rail network to disruption. It could also help to identify which categories of risk have the greatest overall impact on railway network vulnerability, and this may in turn have implications for policy in related areas. It should be noted that the risks (and therefore the criticality levels) identified here will not remain constant over time, but will change in relation to both internal factors, such as rail electrification and centralised control systems) and external factors such as climate change and changing socio-demographic and economic patterns. While the methodology as described here has been developed specifically for the rail network in Great Britain, it could be applied both in other spatial contexts and to other infrastructure systems. In future research the methodology will be applied to multiple infrastructure networks to capture interdependence between rail and other infrastructure systems.

\section{ACKNOWLEDGEMENTS}

The research reported in this paper was part of the UK Infrastructure Transitions Research Consortium (ITRC) funded by the Engineering and Physical Sciences Research Council under Programme Grant EP/I01344X/1. 